\title{
Enhanced diagnostic accuracy for quantitative bone scan using an artificial neural network system: a Japanese multi-center database project
}

Kenichi Nakajima ${ }^{1 *}$, Yasuo Nakajima ${ }^{2}$, Hiroyuki Horikoshi ${ }^{3}$, Munehisa Ueno ${ }^{4}$, Hiroshi Wakabayashi ${ }^{1}$, Tohru Shiga ${ }^{5}$, Mana Yoshimura ${ }^{6}$, Eiji Ohtake ${ }^{7}$, Yoshifumi Sugawara ${ }^{8}$, Hideyasu Matsuyama ${ }^{9}$ and Lars Edenbrandt ${ }^{10}$

\begin{abstract}
Background: Artificial neural network (ANN)-based bone scan index (BSI), a marker of the amount of bone metastasis, has been shown to enhance diagnostic accuracy and reproducibility but is potentially affected by training databases. The aims of this study were to revise the software using a large number of Japanese databases and to validate its diagnostic accuracy compared with the original Swedish training database.

Methods: The BSI was calculated with EXINIbone (EB; EXINI Diagnostics) using the Swedish training database $(n=789)$. The software using Japanese training databases from a single institution (BONENAVI version $1, B N 1$, $n=904$ ) and the revised version from nine institutions (version 2 , BN2, $n=1,532$ ) were compared. The diagnostic accuracy was validated with another 503 multi-center bone scans including patients with prostate $(n=207)$, breast $(n=166)$, and other cancer types. The ANN value (probability of abnormality) and BSI were calculated. Receiver operating characteristic (ROC) and net reclassification improvement (NRI) analyses were performed.
\end{abstract}

Results: The ROC analysis based on the ANN value showed significant improvement from EB to BN1 and BN2. In men $(n=296)$, the area under the curve (AUC) was 0.877 for EB, 0.912 for BN1 ( $p=$ not significant (ns) vs. EB) and 0.934 for BN2 ( $p=0.007$ vs. EB). In women ( $n=207$ ), the AUC was 0.831 for $\mathrm{EB}, 0.910$ for BN1 ( $p=0.016$ vs. EB), and 0.932 for BN2 ( $p<0.0001$ vs. EB). The optimum sensitivity and specificity based on BN2 was $90 \%$ and $84 \%$ for men and $93 \%$ and $85 \%$ for women. In patients with prostate cancer, the AUC was equally high with EB, BN1, and BN2 $(0.939,0.949$, and $0.957, p=n s)$. In patients with breast cancer, the AUC was improved from EB (0.847) to BN1 $(0.910, p=\mathrm{ns})$ and BN2 $(0.924, p=0.039)$. The NRI using ANN between EB and BN1 was $17.7 \%(p=0.0042)$, and that between EB and BN2 was 29.6\% ( $p<0.0001)$. With respect to BSI, the NRI analysis showed downward reclassification with total NRI of $31.9 \%(p<0.0001)$.

Conclusion: In the software for calculating BSI, the multi-institutional database significantly improved identification of bone metastasis compared with the original database, indicating the importance of a sufficient number of training databases including various types of cancers.

Keywords: Bone scintigraphy; Bone scan index; Artificial neural network; Databases; Multi-center study

\footnotetext{
* Correspondence: nakajima@med.kanazawa-u.ac.jp

'Department of Nuclear Medicine, Kanazawa University Hospital, 13-1

Takara-machi, Kanazawa 920-8641, Japan

Full list of author information is available at the end of the article
} 


\section{Background}

Bone scintigraphy has been accepted as a means to identify bone metastases associated with various types of cancer. Even after the advent of single-photon emission computed tomography combined with X-ray computed tomography, whole-body bone imaging is a standard method to survey the existence and extent of bone metastasis. Moreover, although bone scan interpretation may be performed on visual interpretation of wholebody images, an appropriate quantitative approach has been expected. While initial detection of bone metastases is important, quantification of progress of metastasis that results in patients' disability, pain, pathological fractures, and mortality would be also beneficial $[1,2]$. However, there had been no definite imaging method that reflected metastatic disease burden and treatment effect before the advent of bone scan index (BSI) proposed at Memorial Sloan-Kettering Cancer Center [3].

The BSI was developed as a marker of the spread for bone metastasis, which is a fraction of bones involved by a tumor and which realizes the regional distribution of the lesions [4]. The software program for calculating BSI using the neural network system has also been developed using whole-body images with a Swedish database [5]. They successfully applied automatic segmentation of the skeletal regions and automatic detection and feature extraction of hot spots using the neural network system. However, the diagnostic accuracy is potentially influenced by training databases. Whether the same database can be used universally in any study population is yet to be determined. The initial version using a Japanese database showed promising results with a revised database, but it was based on a single-center database [6,7].

The aims of this study were to create a multi-center Japanese database based on a large number of subjects with and without definite bone metastasis and to test the diagnostic accuracy compared with the original European database. In addition, to understand the characteristics of diagnostic accuracy based on the new database, a net reclassification improvement analysis was performed [8].

\section{Methods}

\section{Patients}

The new multi-center training database used in the development of BONENAVI version 2 (BN2) comprised 1,532 patients from nine Japanese hospitals (Table 1). The average age was $68 \pm 10$ (range 20 to 99) years for males and $59 \pm 12$ (range 26 to 91 ) years for females. A total of $42 \%$ of the patients had bone metastasis, with the underlying cancer being prostate cancer in 29\%, breast cancer in $41 \%$, and other cancers in $30 \%$ of the cases. In all hospitals, radiology and/or nuclear medicine specialists made the definitive diagnoses. Every hot spot was classified as metastasis or not, based on information from multiple modalities including X-ray computed tomography (CT), magnetic resonance imaging, positron emission computed tomography, and serial bone scan follow-up studies. All patients had X-ray CT studies, and 1,434 (94\%) patients had two or more bone scans.

Blood sampling including biochemical bone and tumor markers and the patient clinical courses were also used to reach the gold standard classification. Hot spots most likely due to degenerative disease or arthritis, for example, in the vertebrae and shoulder joints, were judged as non-metastatic.

The performance of $\mathrm{BN} 2$ was compared to that of EXINIbone (EB; version 1.3, EXINI Diagnostics AB, Lund, Sweden) and the first version of BONENAVI (BN1; collaboration between EXINI Diagnostics AB and FUJIFILM RI Pharma, Co. Ltd, Tokyo, Japan). The training database of EB comprised 789 bone scans from a single Swedish hospital [9], and that of BN1 comprised 904 bone scans from a single Japanese hospital [7]. The demographics of the patients from the different training databases are shown in Table 1.

A validation group, used to test the performance of $\mathrm{EB}$, $\mathrm{BN} 1$, and BN2, was developed as a second multi-center

Table 1 Demographics of European and Japanese databases and validation groups

\begin{tabular}{|c|c|c|c|c|c|c|}
\hline & \multirow[t]{2}{*}{ EB } & \multirow[t]{2}{*}{ BN1 } & \multirow[t]{2}{*}{ BN2 } & \multicolumn{3}{|c|}{ Validation group } \\
\hline & & & & All & Male & Female \\
\hline N & 789 & 904 & 1,532 & 503 & 296 & 207 \\
\hline Age (years) & $66 \pm 12$ & $64 \pm 12$ & $64 \pm 12$ & $65 \pm 11$ & $68 \pm 9$ & $59 \pm 12$ \\
\hline Male, N (\%) & $508(64)$ & $457(51)$ & $790(52)$ & $296(59)$ & - & - \\
\hline Bone metastases, $N(\%)$ & $262(33)$ & $141(16)$ & $638(42)$ & $169(34)$ & $96(32)$ & $73(35)$ \\
\hline \multicolumn{7}{|l|}{ Types of cancer } \\
\hline Prostate, N (\%) & $425(54)$ & $267(30)$ & $451(29)$ & $207(41)$ & $207(70)$ & - \\
\hline Breast, $N(\%)$ & $217(28)$ & $383(42)$ & $624(41)$ & $166(33)$ & - & $166(80)$ \\
\hline Others, N (\%) & 147 (19) & $254(28)$ & $457(30)$ & $130(26)$ & $89(30)$ & $41(20)$ \\
\hline
\end{tabular}

EB, EXINIbone; BN1, BONENAVI version 1; BN2, BONENAVI version 2. 
group of 503 patients from the same nine Japanese hospitals that participated in the $\mathrm{BN} 2$ training database (Table 1). The classification criteria regarding metastatic diseases were the same as in BN2. The underlying malignancies other than breast cancer and prostate cancer $(n=130)$ were lung cancer $(n=49)$, renal cancer $(n=17)$, esophageal cancer $(n=8)$, gastric cancer $(n=6)$, thyroid cancer $(n=5)$, pancreatic cancer $(n=5)$, and other types of malignancy ( $n=4$ or less for each type).

To accumulate the scintigraphic images for the databases, even though the DICOM data were anonymized, approval of the institutional review board or ethical committee was obtained in all institutions. All the data were accumulated retrospectively. The review boards waived the written informed consent from each patient.

\section{Whole-body bone scan}

Whole-body anterior and posterior images were used for the analysis. A standard dose of 555 to $740 \mathrm{MBq}$ of ${ }^{99} \mathrm{~m}$ Tc-methylene diphosphonate (MDP; FUJIFILM RI Pharma, Co. Ltd, Tokyo, Japan) was injected and imaged $3 \mathrm{~h}$ (range 2.5 to $5.5 \mathrm{~h}$ ) later. The matrix size was $256 \times$ 1,024 . Energy peak was centered at $140 \mathrm{keV}$ with $15 \%$ to $20 \%$ windows.

\section{Automated bone scan analysis}

The automated method for analysis of anterior and posterior whole-body bone scan images has been described previously [9]. Segmentation of the skeleton was performed by fitting an atlas to the patient skeleton using Morphon registration for non-rigid image registration. The atlases were based on 10 normal bone scans from European patients for EB and 23 normal bone scans from Japanese patients for $\mathrm{BN} 1$, whereas gender-specific atlases were developed for BN2 using normal bone scans from 25 male and 25 female Japanese patients. Regions inside the delineated skeleton with intensities exceeding a threshold were defined as hot spots. This threshold varied over different parts of the skeleton and was proportional to the overall intensities found in a neighborhood surrounding each hot spot. This made the algorithm equally sensitive to hot spots in low-intensity regions such as the ribs and high-intensity regions such as the lumbar spine. Each individual hot spot was classified as metastasis or not by an artificial neural network (ANN). Separate ANNs were used for each anatomical region, e.g., skull, spine, ribs, pelvis, and femur, and the different ANNs were used as input for specific sets of variables such as size, shape, intensity, and localization of the hot spot. The training databases for $\mathrm{EB}, \mathrm{BN} 1$, and $\mathrm{BN} 2$ were different as described above, and gender-specific ANNs were developed for BN2. The training of the ANNs was performed using customized software at EXINI Diagnostics. The skeletal involvement of each hot spot was calculated as the percentage of the total skeleton, and the BSI was calculated as the sum of the skeletal involvement of all hot spots classified as metastases by the ANNs.

\section{Statistical analysis}

All the data were expressed as an average and standard deviation. Contingency table analysis was performed to compare values in two groups. The receiver operating characteristic (ROC) analysis was performed and the area under the curve (AUC) was calculated. An optimal cutoff for the sensitivity and specificity could be calculated as the highest value of sensitivity - (1 - specificity). The net reclassification improvement (NRI) analysis for identifying bone metastasis was performed using four ANN groups of 0 to $0.24,0.25$ to $0.49,0.50$ to 0.74 , and 0.75 to 1.00 [8]. In order to evaluate the effect of the software revisions on BSI, the NRI analysis was also performed using four BSI groups of $<0.1,0.1$ to $0.99,1$ to 4.99 , and $\geq 5$. $P$ values $<5 \%$ were considered significant.

\section{Results}

\section{ROC and optimal sensitivity and specificity}

Diagnostic accuracy based on ANN was examined using ROC analysis (Figure 1). Since the databases were made separately for men and women, the ROC AUC based on ANN was calculated in each gender. In men $(n=296)$, the AUC was 0.877, 0.912, and 0.934 for $\mathrm{EB}, \mathrm{BN} 1(p=$ not significant (ns) vs. EB), and BN2 ( $p=0.007$ vs. EB), respectively. The sensitivity was determined as $83 \%$ for $\mathrm{EB}, 88 \%$ for $\mathrm{BN} 1$ ( $p=\mathrm{ns}$ vs. $\mathrm{EB})$, and $90 \%$ for $\mathrm{BN} 2(p=$ ns vs. $E B$ ), whereas specificity was determined as $69 \%$ for $\mathrm{EB}, 83 \%$ for $\mathrm{BN} 1$ ( $p=0.001$ vs. $\mathrm{EB}$ ), and $84 \%$ for $\mathrm{BN} 2$ $(p=0.001$ vs. $\mathrm{EB})$. In women $(n=207)$, the AUC was $0.831,0.910$, and 0.932 for $\mathrm{EB}, \mathrm{BN} 1$ ( $p=0.016$ vs. EB), and $\mathrm{BN} 2(p<0.001$ vs. EB), respectively. The sensitivity was determined as $90 \%$ for $\mathrm{EB}, 81 \%$ for $\mathrm{BN} 1$ ( $p=0.167$ vs. $\mathrm{EB}$ ), and $93 \%$ for $\mathrm{BN} 2$ ( $p=0.774$ vs. $\mathrm{EB})$, whereas the specificity was determined as $51 \%$ for $\mathrm{EB}, 87 \%$ for BN1 $(p<0.001$ vs. $\mathrm{EB})$, and $85 \%$ for $\mathrm{BN} 2(p<0.001$ vs. $\mathrm{EB})$.

Figure 2 shows differences in ROC curves depending on cancer types. When ANN was used to discriminate metastatic patients with both genders combined, the ROC AUC was 0.858 for EB, 0.910 for BN1 $(p=0.067$ vs. $\mathrm{EB})$, and 0.932 for $\mathrm{BN} 2(p<0.0001$ vs. $\mathrm{EB})$. In patients with prostate cancer, the ROC AUC was not improved from EB (0.939), BN1 (0.949), to BN2 (0.957). In patients with breast cancer, however, AUC was improved from $\mathrm{EB}(0.847)$ to $\mathrm{BN} 1(0.910, p=\mathrm{ns})$ and $\mathrm{EB}$ to $\mathrm{BN} 2$ (0.924, $p=0.039)$. In patients with other cancers, AUC was significantly improved from EB $(0.770)$ to $\mathrm{BN} 1$ $(0.861, p=0.023)$ and EB to BN2 $(0.914, p<0.0001)$.

When NRI analys is was performed between $\mathrm{EB}$ and $\mathrm{BN} 2$, net gain in reclassification proportion in patients with metastasis $(n=169)$ was $3.6 \%(p=n s)$, whereas it was $-26.0 \%$ 


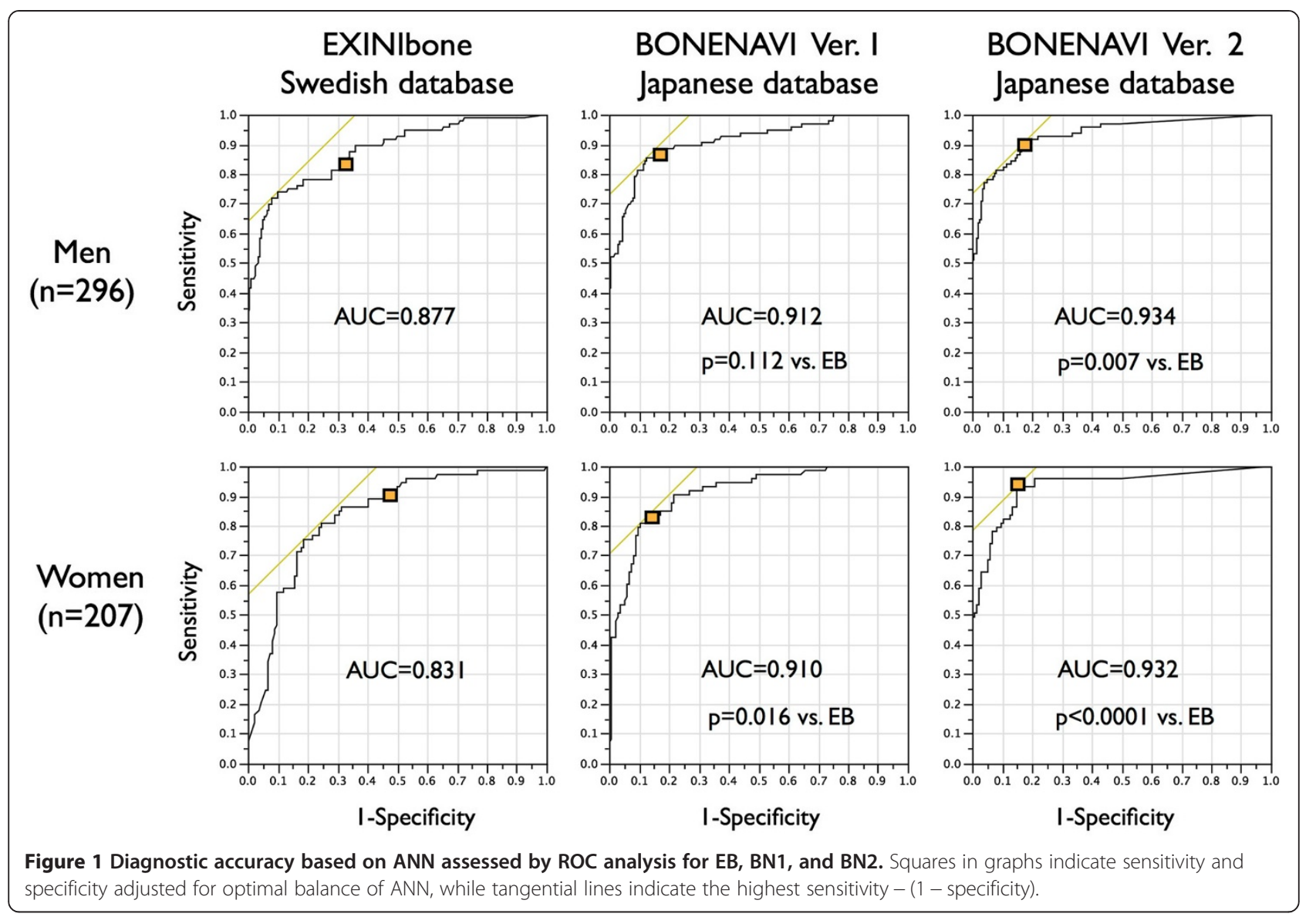

$(p<0.0001)$ in patients without metastasis $(n=334)$ (Table 2). Total NRI was $29.6 \%$ and was highly significant $(p<0.0001)$. The NRI from EB to BN1 was $17.7 \%$ $(p=0.0042)$ with net gains of $-5.3 \%(p=\mathrm{ns})$ and $-23.1 \%$ $(p<0.0001)$ in patients with and without metastasis, respectively. The NRI from $\mathrm{BN} 1$ to $\mathrm{BN} 2$ was $10.4 \%$ $(p=0.064)$ with net gains of $10.1 \%(p=0.020)$ and $-0.3 \%$ $(p=\mathrm{ns})$ in patients with and without metastasis, respectively.

NRI analysis was also performed to evaluate the effect of revision on BSI (Table 3). When EB and BN2 were compared in patients with metastasis, the net gain in reclassification proportion in patients with metastasis was $-40.8 \%(p<0.0001)$. In patients without metastasis, the net gain was $-72.8 \%(p<0.0001)$. The total NRI was $31.9 \%$ and was highly significant $(p<0.0001)$.

Figure 3 shows a patient with prostate cancer with bone metastasis and a patient with breast cancer without bone metastasis. In the patient with prostate cancer, the metastatic lesions were correctly identified by BN2. The breast cancer patient showed a high BSI with EB and a lower BSI in BN1. The BSI was correctly diagnosed as 0 with BN2.

\section{Discussion}

This study was performed as a multi-center project to establish a software program by incorporating a database that includes large number of patients with bone metastasis from various cancer types. While the software based on a Japanese single-center database improved the diagnostic accuracy compared with the software based on the original European database, the multi-center database including 1,532 patients further enhanced the diagnostic accuracy. The large training database also made it possible to use gender-specific analysis in BN2.

In addition to the diagnostic use of the software, BSI provides a quantitative measure that reflects the tumor burden expressed as a percentage of total body skeletal mass. The initial study started at Memorial SloanKettering Cancer Center in patients with prostate cancer and showed good reproducibility and a parallel change with prostate-specific antigen $[3,4]$. BSI has been proved to contain prognostic information in addition to that of conventional prognostic markers such as clinical $\mathrm{T}$ stage, Gleason score, and prostate-specific antigen, and it has therefore drawn the attention of oncologists and urologists [10]. When prostate cancer patients were stratified 


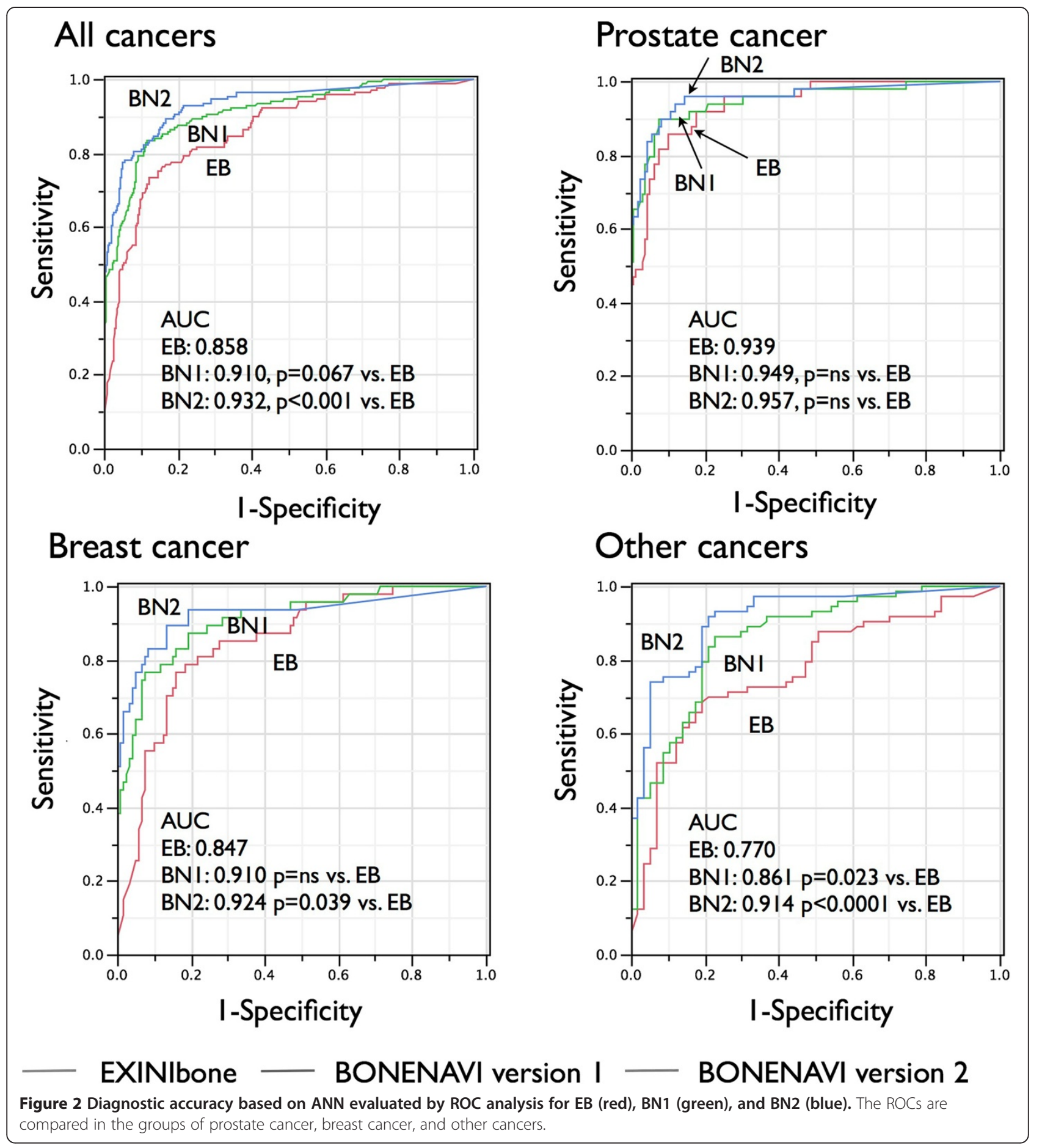

into, for example, high, intermediate, and low BSI groups, significant differences in survival rate were demonstrated [11-13]. On-treatment changes in BSI could be a good response indicator rather than prostatespecific antigen alone in patients with castrationresistant metastatic prostate cancer.

The quantification of bone scans became practical by the use of a computer-aided diagnosis system with
ANN, since the quality of visual bone scan interpretation varied according to readers' experiences [14]. When the segmentation of the skeletons, hot spot detection, evaluation of the characteristics of hot areas, and summed quantitative indexes were available with an automatic method, the reproducibility could be enhanced $[5,15]$. In a study using EB, a close correlation was demonstrated between manual and automated BSI measurements, and 
Table 2 Net reclassification improvement analyses between EB and BN2 based on ANN groups

\begin{tabular}{|c|c|c|c|c|c|c|}
\hline & \multirow[t]{2}{*}{ EB } & \multicolumn{5}{|c|}{ BN2 } \\
\hline & & 0 to 0.24 & 0.25 to 0.49 & 0.50 to 0.74 & 0.75 to 1.00 & Total \\
\hline \multirow{9}{*}{$\begin{array}{l}\text { Metastasis }(n=169) \text { : net gain in reclassification } \\
\text { proportion }=3.6 \%, p=0.38\end{array}$} & 0 to 0.24 & 1 & 3 & 3 & 3 & 10 \\
\hline & & $0.6 \%$ & $1.8 \%$ & $1.8 \%$ & $1.8 \%$ & \\
\hline & 0.25 to 0.49 & 2 & 2 & 4 & 5 & 13 \\
\hline & & $1.2 \%$ & $1.2 \%$ & $2.4 \%$ & $3.0 \%$ & \\
\hline & 0.50 to 0.74 & 2 & 1 & 5 & 8 & 16 \\
\hline & & $1.2 \%$ & $0.6 \%$ & $3.0 \%$ & $4.7 \%$ & \\
\hline & 0.75 to 1.00 & 1 & 3 & 11 & 115 & 130 \\
\hline & & $0.6 \%$ & $1.8 \%$ & $6.5 \%$ & $68.0 \%$ & \\
\hline & Total & 6 & 9 & 23 & 131 & 169 \\
\hline \multirow{9}{*}{$\begin{array}{l}\text { No metastasis }(n=334) \text { : net gain in reclassification } \\
\text { proportion }=-26.0 \%, p<0.0001\end{array}$} & 0 to 0.24 & 96 & 31 & 14 & 4 & 145 \\
\hline & & $28.7 \%$ & $9.3 \%$ & $4.2 \%$ & $1.2 \%$ & \\
\hline & 0.25 to 0.49 & 41 & 14 & 5 & 2 & 62 \\
\hline & & $12.3 \%$ & $4.2 \%$ & $1.5 \%$ & $0.6 \%$ & \\
\hline & 0.50 to 0.74 & 34 & 19 & 9 & 5 & 67 \\
\hline & & $10.2 \%$ & $5.7 \%$ & $2.7 \%$ & $1.5 \%$ & \\
\hline & 0.75 to 1.00 & 35 & 11 & 8 & 6 & 60 \\
\hline & & $10.5 \%$ & $3.3 \%$ & $2.4 \%$ & $1.8 \%$ & \\
\hline & Total & 206 & 75 & 36 & 17 & 334 \\
\hline
\end{tabular}

Total NRI $=29.6 \%, p<0.0001$.

Table 3 Net reclassification improvement analyses between EB and BN2 based on BSI groups

\begin{tabular}{|c|c|c|c|c|c|c|}
\hline & \multirow[t]{2}{*}{$\mathrm{EB}$} & \multicolumn{5}{|c|}{ BN2 } \\
\hline & & $<0.1$ & 0.1 to 1 & 1 to 5 & $>5$ & Total \\
\hline \multirow{9}{*}{$\begin{array}{l}\text { Metastasis }(n=169) \text { : net gain in reclassification } \\
\text { proportion }=-40.8 \%, p<0.0001\end{array}$} & $<0.1$ & 3 & 0 & 0 & 0 & 3 \\
\hline & & $1.8 \%$ & $0.0 \%$ & $0.0 \%$ & $0.0 \%$ & \\
\hline & 0.1 to 1 & 6 & 28 & 0 & 0 & 34 \\
\hline & & $3.6 \%$ & $16.6 \%$ & $0.0 \%$ & $0.0 \%$ & \\
\hline & 1 to 5 & 4 & 48 & 40 & 0 & 92 \\
\hline & & $2.4 \%$ & $28.4 \%$ & $23.7 \%$ & $0.0 \%$ & \\
\hline & $>5$ & 0 & 1 & 10 & 29 & 40 \\
\hline & & $0.0 \%$ & $0.6 \%$ & $5.9 \%$ & $17.2 \%$ & \\
\hline & Total & 13 & 77 & 50 & 29 & 169 \\
\hline \multirow{9}{*}{$\begin{array}{l}\text { No metastasis }(n=334) \text { net gain in reclassification } \\
\text { proportion }=-72.8 \%, p<0.0001\end{array}$} & $<0.1$ & 43 & 4 & 0 & 0 & 47 \\
\hline & & $25.4 \%$ & $2.4 \%$ & $0.0 \%$ & $0.0 \%$ & \\
\hline & 0.1 to 1 & 129 & 31 & 0 & 0 & 160 \\
\hline & & $76.3 \%$ & $18.3 \%$ & $0.0 \%$ & $0.0 \%$ & \\
\hline & 1 to 5 & 63 & 38 & 9 & 0 & 110 \\
\hline & & $37.3 \%$ & $22.5 \%$ & $5.3 \%$ & $0.0 \%$ & \\
\hline & $>5$ & 7 & 7 & 3 & 0 & 17 \\
\hline & & $4.1 \%$ & $4.1 \%$ & $1.8 \%$ & $0.0 \%$ & \\
\hline & Total & 242 & 80 & 12 & 0 & 334 \\
\hline
\end{tabular}

Total NRI $=31.9 \%, p<0.0001$. 

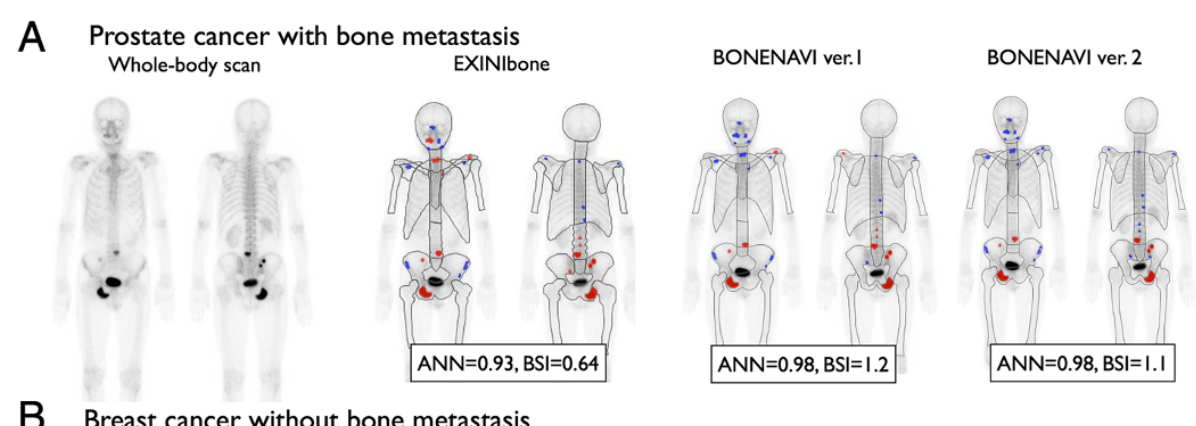

B Breast cancer without bone metastasis
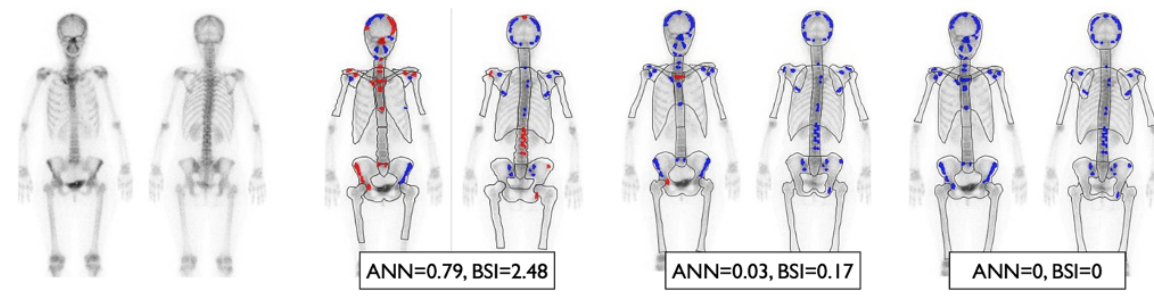

Figure 3 A 69-year-old man with prostate cancer (A) and a 53-year-old woman with breast cancer (B). The patient with prostate cancer had multiple metastases that were correctly identified by BN2, and the BSI was increased with BN2 compared with EB. The patient with breast cancer did not have metastasis, and both ANN and BSI were reduced by the revised versions with Japanese training databases. Red hot spots denote high-risk lesions, namely high probability of metastases, whereas blue hot spots denote low-risk lesions.

the merit of the latter was $100 \%$ reproducibility [9]. Owing to simple application, BSI could be incorporated into clinical practice, while patients were diagnosed, treated, and followed up on.

Training databases are essential for a neural network system to diagnose bone metastases. In this study, we used only Japanese patients with definite diagnosis for the existence of bone metastasis. In addition to patient-based diagnostic accuracy, all the hot areas were confirmed by other imaging modalities and/or follow-up bone scans. Since the BN1 included only 141 (16\%) patients with bone metastases from one hospital, it was increased to 638 (42\%) patients from nine hospitals. The number of hot spots in ribs, for example, was increased from 2,303 (metastasis 50\%) in $\mathrm{BN} 1$ to 3,294 (metastasis $43 \%$ ) in BN2, which contributed to enhancing the learning volumes. When it is utilized in a number of hospitals, the multi-center database judged by multiple experts would be beneficial for enhancing diagnostic accuracy in computer learning.

The larger collection of databases including various cancer types is essential for obtaining appropriate BSI values. When we used the EB on Japanese patients for the first time, hot spots indicating high probability of abnormality were frequently noticed in the skull, shoulder joints, and lumbar vertebrae. These regions included diffuse metabolic accumulation in the skull of female patients and degenerative changes in the vertebrae and joints. About half $(n=425)$ of the Swedish database was from prostate cancer and 28\% $(n=217)$ from breast cancer. In contrast, the Japanese databases for BN2 included $29 \%(n=451)$ from prostate cancer, $41 \%(n=624)$ from breast cancer, and 30\% $(n=457)$ from other cancer types. The BN2 databases, therefore, included various cancer types and were closer to the usual clinical environment. From the viewpoint of Japanese populationspecific databases, not only the physical stature but also the incidence of degenerative or deformative bone changes might differ between Swedish and Japanese subjects. When EB and BN1 were compared, NRI analysis with ANN showed that BN1 increased negative cases in patients without metastasis, indicating significantly decreased false-positive cases. BN2 further adjusted the diagnostic accuracy and reclassified the metastatic lesions into the higher ANN groups. With respect to the influence of revisions on BSI, the NRI analysis showed that reclassification was downward in both metastatic and non-metastatic groups. However, reclassification of non-metastatic patients into the lower risk BSI seemed to have meaning, and total net reclassification was improved in one third of the patients. The final effect of revision on predicting prognosis should be confirmed in future follow-up studies.

Notable effects of training databases differ among prostate, breast, and other cancers. The differences among cancer types seemed to be related to osteoblastic and osteolytic activity of the bone metastases and their imbalance in regulation [16]. Quantitative measurement of bone metastasis or BSI has most widely been used in patients with prostate cancer $[9,10,12,17,18]$. Prostate cancer shows typical osteoblastic metastasis based on radiological findings, though it is also associated with osteoclastic process and bone resorption. The bone scan 
appearance in prostate cancer reveals multiple hot spots and even the so-called superscan in extreme situations. Detecting all metastatic hot areas is important when demanding an overview of the whole amount of metastasis in prostate cancer. Therefore, the diagnostic accuracy in identifying bone metastasis was high even with $\mathrm{EB}$, and further improvement by BN1 and BN2 was not achieved. In contrast, breast cancer commonly metastasizes to bones and destroys its structure, which causes both osteolytic and osteoblastic appearance in bones. The bone scan might show relatively mild activity or even cold areas in the pure osteolytic lesions. Higher fractions of breast and other cancer types in $\mathrm{BN} 2$ as compared with EB were also noted, namely, non-prostate cancer, $46 \%(n=364)$ for $\mathrm{EB}, 70 \%(n=637)$ for $\mathrm{BN} 1$, and $71 \%$ $(n=1081)$ for BN2 (Table 1). To enhance the diagnostic accuracy in breast cancer metastasis, decreasing falsepositive hot spots had practically important meaning, and it explained why the diagnostic improvement was obtained in BN1 and BN2 as compared with EB.

\section{Limitations}

The detection of metastasis was based on the hot areas, and cold lesions were not included for training the ANN system. However, since most of the diagnosis of the bone metastasis was made by the accumulation of ${ }^{99 \mathrm{~m}} \mathrm{Tc}-\mathrm{MDP}$, the utility of BSI would not be substantially changed. Although database training was performed using all subjects, specific cancer type-based training, for example, prostate cancer-specific and breast cancer-specific training databases, could be applied. This process requires considerable time for separate training and will be studied in future works. Finally, when the NRI analysis is performed based on skeletal-related events, instead of diagnosis of metastases, true values of BSI will be confirmed in the future.

Even when we consider the possibility of ${ }^{18} \mathrm{~F} \mathrm{NaF}$ positron emission computed tomography in the future, a similar approach using ANN and new training databases might be an interesting project. What kinds of algorithm of ANN system are appropriate for tomographic images and/or maximum intensity projection images should be investigated.

\section{Conclusion}

The Japanese multi-center database significantly improved the diagnostic accuracy, showing AUC of 0.93 in both genders with ANN. The improvement from EB to $\mathrm{BN} 1$ to BN2 was particularly high in patients with breast cancer and other cancer types, while the diagnostic accuracy was equally high in patients with prostate cancer. Reclassification analysis showed that the main improvement was the decrease of false-positive results and that non-metastatic patients were reclassified into lower BSI groups. A large number of training databases including various cancer types were effective in improving the diagnostic accuracy.

\section{Competing interests}

LE is employed by and a shareholder of EXINI Diagnostics AB. KN has a collaborative research with FUJIFILM RI Pharma, Co. Ltd, Japan. While FUJIFILM RI Pharma is involved in the distribution of BONENAVI software in Japan, KN has no relationship relevant to the contents of this study to disclose.

\section{Authors' contributions}

KN drafted the manuscript and LE and HH edited it. All the authors participated in the study design, and all but LE were involved in the final diagnosis and interpretation of bone scans. KN performed the statistical analysis of the data and YN confirmed it. All authors read and approved the final manuscript.

\section{Acknowledgements}

This work was performed as a multi-center study for creating a new database involving nine institutions and supported by the cooperation of medical doctors and technologists in all institutions. Within the multi-center study, the authors would like to express their gratitude for the cooperation of Dr. Tsuyoshi Kawano (Kanagawa Cancer Center); Dr. Kiichiro Kodaira (International Medical Centre-Comprehensive Cancer Centre, Saitama Medical University); Takeshi Ono, RT (Shikoku Cancer Center); Dr. Koichiro Tsugawa, Dr. Istuko Okuda, Dr. Yasuyuki Kojima, and Yoshiaki Maehara, RT (St. Marianna University School of Medicine); Dr. Kiyoshi Koizumi, Dr. Tsuyoshi Hashimoto, Dr. Kunihito Suzuki, and Kenji Uchida, RT (Tokyo Medical University); Dr. Osamu Manabe (Hokkaido University); and Dr. Kazuhiro Nagao, Dr. Osamu Tokuda, and Yona Oishi, RT (Yamaguchi University). The authors wish to thank Mr. Mattias Ohlsson and Mr. Jens Richter (EXINI Diagnostics, Lund, Sweden), who were involved in software development and training with databases. The authors also thank Mr. Kazunori Kawakami and Mr. Akihiro Kikuchi (FUJIFILM RI Pharma, Co. Ltd, Tokyo, Japan) for their assistance in accumulating all the databases and for their valuable suggestions. They also thank Mr. Ronald Belisle for his editorial assistance and preparation of the manuscript.

\section{Author details}

'Department of Nuclear Medicine, Kanazawa University Hospital, 13-1 Takara-machi, Kanazawa 920-8641, Japan. ${ }^{2}$ Department of Radiology St. Marianna University School of Medicine, Kawasaki 216-8511, Japan. ${ }^{3}$ Department of Diagnostic Radiology, Gunma Prefectural Cancer Center, Ota 373-8550, Japan. ${ }^{4}$ Department of Urology, International Medical Centre-Comprehensive Cancer Centre, Saitama Medical University, Kawagoe 350-1298, Japan. ${ }^{5}$ Department of Nuclear Medicine, Hokkaido University Graduate School of Medicine, Sapporo 060-8638, Japan. ${ }^{6}$ Department of Radiology, Tokyo Medical University, Tokyo 160-8402, Japan. ${ }^{7}$ Department of Nuclear Medicine, Kanagawa Cancer Center, Yokohama 241-8515, Japan. ${ }^{8}$ Department of Diagnostic Radiology, Shikoku Cancer Center, Matsuyama 791-0280, Japan. ${ }^{9}$ Department of Urology, Yamaguchi University School of Medicine, Yamaguchi 755-8505, Japan. ${ }^{10}$ Department of Clinical Physiology and Nuclear Medicine, Gothenburg University, Gothenburg 41345, Sweden.

Received: 11 October 2013 Accepted: 10 December 2013 Published: 26 December 2013

\section{References}

1. Soloway MS, Hardeman SW, Hickey D, Raymond J, Todd B, Soloway S, Raymond J, Moinuddin M: Stratification of patients with metastatic prostate cancer based on extent of disease on initial bone scan. Cancer 1988, 61:195-202.

2. Noguchi M, Kikuchi $H$, Ishibashi M, Noda S: Percentage of the positive area of bone metastasis is an independent predictor of disease death in advanced prostate cancer. Br J Cancer 2003, 88:195-201.

3. Erdi YE, Humm JL, Imbriaco M, Yeung H, Larson SM: Quantitative bone metastases analysis based on image segmentation. J NuCl Med 1997, 38:1401-1406.

4. Imbriaco M, Larson SM, Yeung HW, Mawlawi OR, Erdi Y, Venkatraman ES, Scher HI: A new parameter for measuring metastatic bone involvement 
by prostate cancer: the Bone Scan Index. Clin Cancer Res 1998, 4:1765-1772.

5. Sadik M, Hamadeh I, Nordblom P, Suurkula M, Hoglund P, Ohlsson M, Edenbrandt L: Computer-assisted interpretation of planar whole-body bone scans. J Nucl Med 2008, 49:1958-1965.

6. Kikuchi A, Onoguchi M, Horikoshi H, Sjostrand K, Edenbrandt L: Automated segmentation of the skeleton in whole-body bone scans: influence of difference in atlas. Nucl Med Commun 2012, 33:947-953.

7. Horikoshi H, Kikuchi A, Onoguchi M, Sjostrand K, Edenbrandt L: Computer-aided diagnosis system for bone scintigrams from Japanese patients: importance of training database. Ann Nucl Med 2012, 26:622-626.

8. Pencina MJ, D'Agostino RB Sr, D'Agostino RB Jr, Vasan RS: Evaluating the added predictive ability of a new marker: from area under the ROC curve to reclassification and beyond. Stat Med 2008, 27:157-172. discussion 207-12.

9. Ulmert D, Kaboteh R, Fox JJ, Savage C, Evans MJ, Lilja H, Abrahamsson PA, Björk T, Gerdtsson A, Bjartell A, Gjertsson P, Höglund P, Lomsky M, Ohlsson M, Richter J, Sadik M, Morris MJ, Scher HI, Sjöstrand K, Yu A, Suurküla M, Edenbrandt L, Larson SM: A novel automated platform for quantifying the extent of skeletal tumour involvement in prostate cancer patients using the Bone Scan Index. Eur Urol 2012, 62:78-84.

10. Scher HI, Morris MJ, Larson S, Heller G: Validation and clinical utility of prostate cancer biomarkers. Nat Rev Clin Oncol 2013, 10:225-234.

11. Sabbatini P, Larson SM, Kremer A, Zhang ZF, Sun M, Yeung H, Imbriaco M, Horak I, Conolly M, Ding C, Ouyang P, Kelly WK, Scher HI: Prognostic significance of extent of disease in bone in patients with androgenindependent prostate cancer. J Clin Oncol 1999, 17:948-957.

12. Dennis ER, Jia X, Mezheritskiy IS, Stephenson RD, Schoder H, Fox JJ, Heller G, Scher HI, Larson SM, Morris MJ: Bone scan index: a quantitative treatment response biomarker for castration-resistant metastatic prostate cancer. J Clin Oncol 2012, 30:519-524.

13. Kaboteh R, Damber JE, Gjertsson P, Hoglund P, Lomsky M, Ohlsson M, Edenbrandt L: Bone Scan Index: a prognostic imaging biomarker for high-risk prostate cancer patients receiving primary hormonal therapy. EJNMMI Res 2013, 3:9.

14. Sadik M, Suurkula M, Hoglund P, Jarund A, Edenbrandt L: Quality of planar whole-body bone scan interpretations-a nationwide survey. Eur J NuCl Med Mol Imaging 2008, 35:1464-1472.

15. Sadik M, Suurkula M, Hoglund P, Jarund A, Edenbrandt L: Improved classifications of planar whole-body bone scans using a computerassisted diagnosis system: a multicenter, multiple-reader, multiple-case study. J Nucl Med 2009, 50:368-375.

16. Guise TA, Mohammad KS, Clines G, Stebbins EG, Wong DH, Higgins LS, Vessella R, Corey E, Padalecki S, Suva L, Chirgwin JM: Basic mechanisms responsible for osteolytic and osteoblastic bone metastases. Clin Cancer Res 2006, 12:6213s-6216s.

17. Fox JJ, Morris MJ, Larson SM, Schoder H, Scher HI: Developing imaging strategies for castration resistant prostate cancer. Acta Oncol 2011, 50(Suppl 1):39-48.

18. Kelly WK, Halabi S, Carducci M, George D, Mahoney JF, Stadler WM, Morris M, Kantoff P, Monk JP, Kaplan E, Vogelzang NJ, Small EJ:

Randomized, double-blind, placebo-controlled phase III trial comparing docetaxel and prednisone with or without bevacizumab in men with metastatic castration-resistant prostate cancer: CALGB 90401. J Clin Oncol 2012, 30:1534-1540.

doi:10.1186/2191-219X-3-83

Cite this article as: Nakajima et al.: Enhanced diagnostic accuracy for quantitative bone scan using an artificial neural network system: a Japanese multi-center database project. EJNMMI Research 2013 3:83.

\section{Submit your manuscript to a SpringerOpen ${ }^{\odot}$ journal and benefit from:}

- Convenient online submission

- Rigorous peer review

- Immediate publication on acceptance

- Open access: articles freely available online

- High visibility within the field

- Retaining the copyright to your article

Submit your next manuscript at $>$ springeropen.com 